Fardella, C., Jiménez Vargas, F., Rivera-Vargas, P. y Baleriola, E. (2022). Salir de la sombra. Una revisión sistemática sobre shadowing como propuesta metodológica para la investigación educativa. Revista de Investigación Educativa, 40(1), 257-274.

DOI: http://dx.doi.org/10.6018/rie.464151

\title{
Salir de la sombra. Una revisión sistemática sobre shadowing como propuesta metodológica para la investigación educativa
}

\section{Coming out of the shadow. A systematic review of shadowing as a methodological approach to educational research}

\author{
Carla Fardella*, Felipe Jiménez Vargas**, Pablo Rivera-Vargas ${ }^{1 * * * y *}$ y Enrique Baleriola**** \\ * Universidad Andrés Bello (Chile) \\ ** Universidad de las Américas (Chile) \\ *** Universidad de Barcelona (España) \\ **** Universitat Oberta de Catalunya (España)
}

\begin{abstract}
Resumen
Cuando hablamos de investigación educativa, hacemos referencia a un campo de conocimiento disputado por múltiples disciplinas y organismos internacionales, que pueden dejar en la sombra a los actores escolares. El shadowing es una estrategia etnográfica relativamente novedosa en los entornos académicos de habla hispana, que justamente emerge con la intención de acentuar el valor que tiene para la investigación educativa, la observación de contextos y comunidades específicas. El objetivo del presente artículo es dar a conocer cuáles son las principales características, posibilidades y limitaciones del shadowing para la investigación educativa. A partir de un enfoque cualitativo, se ha llevado a cabo una revisión de literatura de 37 artículos científicos que describen y reportan resultados sobre su uso. Los resultados reportan (1) origen, ámbitos de aplicación y caracterización, (2) experiencias y usos de la estrategia y (3) las implicancias ético-pragmáticas de la estrategia. Adicionalmente, se plantean algunas reflexiones finales sobre su utilidad y validez a la hora de generar conocimiento educativo focalizado en lo local.

Palabras clave: investigación social, etnografía, observación, escuela, docente.
\end{abstract}

1 Correspondencia: Pablo Rivera-Vargas, pablorivera@ub.edu. Universidad de Barcelona (España). 


\begin{abstract}
When we talk about educational research, we refer to a field of knowledge disputed by multiple disciplines and international organizations, which can cast a shadow on school actors. Shadowing is a relatively new ethnographic strategy in Spanish-speaking academic environments, that precisely emerges in order to highlight the importance of the observation of specific contexts and communities for educational research. Thus, the objective of this article is to show the main characteristics, possibilities, and limitations of shadowing for educational research. From a qualitative approach, a literature review of 37 scientific articles describing and reporting results on its use has been carried out. The reported findings are organized into three categories: (1) its origin, application areas and characterization, (2) experiences and uses of the strategy and (3) its ethical-pragmatic implications. Additionally, some final reflections on its usefulness and validity for generating localized educational knowledge are raised.
\end{abstract}

Keywords: social research, ethnography, observation, school, teacher.

\title{
Introducción
}

Como es sabido existen diversas tradiciones en la investigación educativa, aunque no todas han gozado históricamente hablando, de la misma legitimidad y reconocimiento. Si bien contamos con el despliegue de una importante tradición critica que ha tensionado permanentemente el campo educativo (Knutas, 2019; Sancho-Gil et al., 2020), en las últimas décadas han proliferado las miradas estructurales, estandarizadas y supra - institucionales para estudiar y construir lo educativo (Biesta, 2015; Hammersley, 2018). Junto a esto, el actual sistema de patrocinio y financiamiento de la investigación ha impreso una demanda creciente de utilidad, impacto y transferencia del conocimiento, que privilegia y superpone el estudio de las condiciones objetivas y medibles que presenta el sistema educativo en su conjunto para asignar presupuestos, monitorear el éxito/fracaso de las reformas educativas, así como para el análisis comparado entre países (Biesta, 2015; Bøe et al., 2016; Bueger y Gadinger, 2018; Fardella, 2020; Hammersley, 2018; World Bank, 2011).

Más allá de las resistencias y los posicionamientos críticos, una parte significativa de la investigación educativa actual está al servicio de las necesidades de producción de información estandarizada que tiende a pasar por alto aspectos locales e idiosincráticos de las comunidades educativas y sus actores (Erstad et al., 2021; Ward y Delamont, 2020). Al respecto, diferentes estudios advierten sobre la creciente distancia entre la realidad relatada por la investigación educativa y la realidad vivida por las comunidades educativas (Farley-Ripple, et al., 2018; Jiménez et al., 2017; Sancho-Gil et al., 2020). Este enfoque ha tendido a sobreponer los métodos y miradas epistémicas de disciplinas tales como la economía, la sociología o la psicología, subordinando el saber y los métodos idiosincráticos del campo de la educación. De ahí que el saber educativo tenga una débil incidencia en la actual construcción de políticas públicas educativas, sumado a un saber docente desacreditado y condicionado por el denominado "saber experto" (Fardella, 2020). La frágil legitimidad y autonomía conferida a la investigación educativa no solo deja en evidencia una inercia que favorece un modelo único y privilegiado de saber científico, sino que propicia además la omisión y silenciamiento 
de docentes y otros actores escolares que día a día construyen y sostienen las comunidades educativas (Erstad et al., 2021).

Como consecuencia de lo expuesto, este trabajo promueve una investigación educativa idiosincrática que, a partir de las necesidades locales, pueda construir sus propios fenómenos, problemas y estrategias metodológicas para generar conocimiento. Con este propósito como horizonte, relevamos la etnografía escolar y en particular del shadowing [sombreado] a partir de la pregunta que guía nuestro trabajo: ¿en qué consiste la estrategia de sombreado y cómo ésta puede fomentar la autonomía de la investigación educativa? Para responder esta pregunta, se reportan los resultados obtenidos del análisis a 38 artículos científicos sobre la estrategia mencionada publicados entre el año 1975 y 2020. Los resultados se presentan a partir de tres categorías emergentes: 1) Origen, ámbitos de aplicación y caracterización de la estrategia; 2) Experiencias en investigación y usos de la estrategia; 3) Implicaciones ético-pragmáticas de la estrategia.

De esta manera se espera contribuir en la construcción de un campo de producción de conocimiento educativo que puede ser agenciado por los y las docentes, desde posiciones teóricas, técnicas y políticas ancladas en el trabajo educativo, con la consecuente legitimidad y autonomía.

\section{El método etnográfico en la investigación educativa}

El método etnográfico se posiciona en contraste a la investigación positivista a partir de una sensibilidad hacia lo local e idiosincrático de las comunidades o perspectivas "nativas" (Delamont y Atkinson, 2018). La etnografía es un método ampliamente usado y por lo mismo no siempre hay consenso en su definición (Hammersley, 2018). Más allá de las diferencias, una cualidad consensuada es el énfasis en la descripción profunda de una comunidad o cultura a partir de la observación, recolección de documentos, así como conversaciones durante un periodo prolongado (Contreras et al., 2016).

Particularmente, la etnografía educativa otorga centralidad a los procesos, significados y prácticas de las comunidades educativas, cómo son las relaciones y las dinámicas cotidianas que sostienen lo escolar (Beach et al., 2018). Por lo mismo, algunas estrategias del método deben ser adaptadas a las cualidades específicas del contexto. De acuerdo con Contreras et al (2016) la etnografía en contextos escolares debe poner atención a aspectos tales como la posición del/a etnógrafo/a o la negociación de la entrada al campo dada la jerarquía y la cultura de evaluación que permea estas instituciones.

Es posible subrayar que el enfoque etnográfico promueve el saber de las comunidades escolares y la autonomía de la investigación educativa principalmente a través de 3 aspectos que desarrollaremos a continuación, a saber: (i) el énfasis en la descripción y comprensión de lo local; (ii) la relación simétrica con actores y actoras locales en la construcción de conocimiento; y (iii) la atención a elementos tradicionalmente no estudiados de la cultura escolar (Beach et al., 2018; Contreras et al., 2016; Gorup, 2016; Ward y Delamont, 2020).

En primer lugar, si bien el énfasis en la descripción y comprensión de las perspectivas nativas por sobre el poder de predicción es una cualidad importante de los enfoques cualitativos, para el método etnográfico se trata de un aspecto constitutivo (Hammersley, 2018). Pese a que el objeto y los modos de hacer etnografía han ido cambiado a lo largo 
de las últimas décadas, comprender de primera fuente y desde dentro de la comunidad es algo que mantiene su protagonismo en el tiempo (Beach et al., 2018). Como señalan Hamersley y Atkinson (2005), el propósito de toda investigación etnográfica es la producción de conocimiento a través de las descripciones profundas de la cultura estudiada. No obstante, el trabajo etnográfico no sólo provee descripciones profundas que permiten comprender la dinámica escolar desde la óptica de los nativos y nativas, sino también la transformación de la propia realidad educativa desde la reflexividad de sus propios actores y actoras. Se trata de un método, por tanto, clave para que todos los actores de la educación puedan participar legítimamente en la construcción de su conocimiento disciplinar (Gorup, 2016).

En segundo lugar, la construcción de un conocimiento disciplinar propio de la escuela debe emerger necesariamente como resultado de una relación simétrica entre etnógrafos/as y nativos/as. Es así como la participación prolongada de los etnógrafos y etnógrafas en el contexto escolar facilita establecer una relación generativa de reflexiones que permiten reconstruir y reestablecer la realidad estudiada a partir del dialogo y la experiencia conjunta (Contreras et al., 2016).

En este sentido, el protagonismo de las comunidades locales, en este caso docentes, es crucial para la generación de conocimiento (Baleriola, 2018). Esto ofrece una ventaja clara: el o la docente que hace etnografía es autor/a de la realidad que acontece en su escuela, pues tiene una idea formada (más o menos explícita) acerca de por qué ocurre un problema en concreto, o qué elementos están impidiendo el normal devenir de la enseñanza. Esto no quita que por otro lado se estén corriendo ciertos riesgos al ser un/a etnógrafo/a que forma parte del propio problema investigado. Por ejemplo, el o la docente pueden tener tan asimiladas ciertas explicaciones o comportamientos que los dé por hecho e ignoren que son producto de una forma particular de hacer las cosas, naturalizando su devenir. En este sentido, la utilidad de la etnografía en investigación educativa es la posibilidad de ir construyendo teorías y conceptos sobre cualquier aspecto escolar desde una base disciplinar magisterial, y no necesariamente psicológica, sociológica, o económica.

En tercer lugar, el método etnográfico permite documentar lo no documentado (Denzin y Lincoln, 2018), y al mismo tiempo aportar evidencias para complejizar lo documentado. A su vez, el énfasis en la descripción implica atender no solo aspectos simbólicos y humanos, sino también infraestructura de la cultura. Las miradas posthumanistas, la Teoría del Actor-Red o bien la Epistemología Feminista, aun siendo divergentes en múltiples aspectos, comparten una serie de supuestos donde la atención a los elementos materiales son condición para comprender en profundidad lo social. En este sentido, la etnografía en sus diversas formas es una herramienta esencial para lograrlo (Sisto y Zelaya, 2013).

Aun cuando la dimensión simbólica en la comunidad a estudiar es importantísima para la experiencia etnográfica, existen otros elementos como las materialidades y los objetos que pueden ser decisivos a la hora de comprender la cultura de una comunidad (Hammersley, 2018; Sisto y Zelaya, 2013; Czarniawska, 2018a). Ilustración de ellos son las políticas escolares, los instrumentos de gestión escolar, los recursos didácticos, la infraestructura del patio o el aula así como la arquitectura de las escuelas, todos elementos que están configurando sistemáticamente lo que es cada escuela y lo que en ella ocurre. 
En síntesis, la etnografía educativa es un campo en desarrollo, especialmente desafiado por el actual contexto de organización de la ciencia (Hammersley, 2018; Fardella, 2020), así como por la historia de investigación educativa (Delamont, S. y Atkinson, 2018; Ward y Delamont, 2020). Es en este escenario que el shadowing se constituye como una estrategia pertinente y valiosa para potenciar, tanto la etnografía escolar como para superar los desafíos históricos y contemporáneos de la investigación educativa.

\section{Método}

Nuestro trabajo es el resultado de un ejercicio de revisión sistemática de literatura (Strauss y Corbin, 2016; Bru et al., 2020). Se trata de la revisión de estudios recapitulados o investigaciones bibliográficas sobre un tema determinado, en las que se reúnen, analizan y discuten informaciones previamente publicadas. Su principal utilidad es la de sintetizar y sistematizar literatura científica fragmentada. Esta estrategia organiza la literatura buscando responder una interrogante específica, que mediante su reorganización nos permita generar conocimiento específico sobre un tema (Cooper, et al., 2018). Para su correcta elaboración, en este trabajo se han seguido las directrices propuestas en la declaración PRISMA (Urrútia y Bonfill, 2010; Bru et al., 2020) para la realización de revisiones sistemáticas (Figura 1).

\section{Diseño y procedimiento}

Se identificaron y analizaron diferentes artículos publicados en revistas científicas online, a partir de la consulta llevada a cabo en las bases de datos WOS $^{2}$, SCOPUS ${ }^{3}$, SCIELO $^{4}$ y GOOGLE SCHOLAR ${ }^{5}$. Los términos de búsqueda incluyeron diferentes combinaciones de las siguientes palabras: shadowing, seguimiento, sombreamiento, revisión, educación, investigación, etnografía escolar; seguidas del operador boleano "AND". En paralelo a esto, se trabajó bajo la técnica de "bola de nieve" (Strauss y Corbin, 2016), seleccionando directamente los artículos relevantes que funcionaban como referencia para los trabajos ya encontrados, hasta llegar al momento de saturación muestral (Delamont y Atkinson; 2018). De este procedimiento se obtuvieron 220 artículos.

Para el primer filtro, se procedió a la lectura de los resúmenes, descartando aquellos que se alejaban de la temática sustantivamente, no ocupaban la técnica de shadowing, se duplicaban o estaban en un idioma distinto al español, inglés, portugués o francés; con este primer filtro se obtuvieron 87 trabajos. Para el segundo filtro, se hizo una lectura de los artículos cribados, descartando aquellos a los que no se pudo acceder, y aquellos donde la temática del shadowing aparecía en el artículo, pero no era el tema central; con este segundo filtro se obtuvieron 60 trabajos. Posteriormente, en el tercer filtro, se hizo una lectura profunda utilizando el software Atlas.Ti para agrupar los estudios en base a su temática, ámbito, y tipo de estudio realizado. Finalmente, tal

2 Disponible en: www.webofknowledge.com

3 Disponible en: https://www.scopus.com/

4 Disponible en: https://www.scielo.org/es/

5 Esta herramienta fue utilizada esencialmente para la búsqueda de libros sobre el tema. Disponible en: https://scholar.google.com/ 
como se puede observar en la tabla 1 , se obtuvieron un total de 37 artículos entre los años 1975 y 2020 (cuarto filtro) (Figura 1).

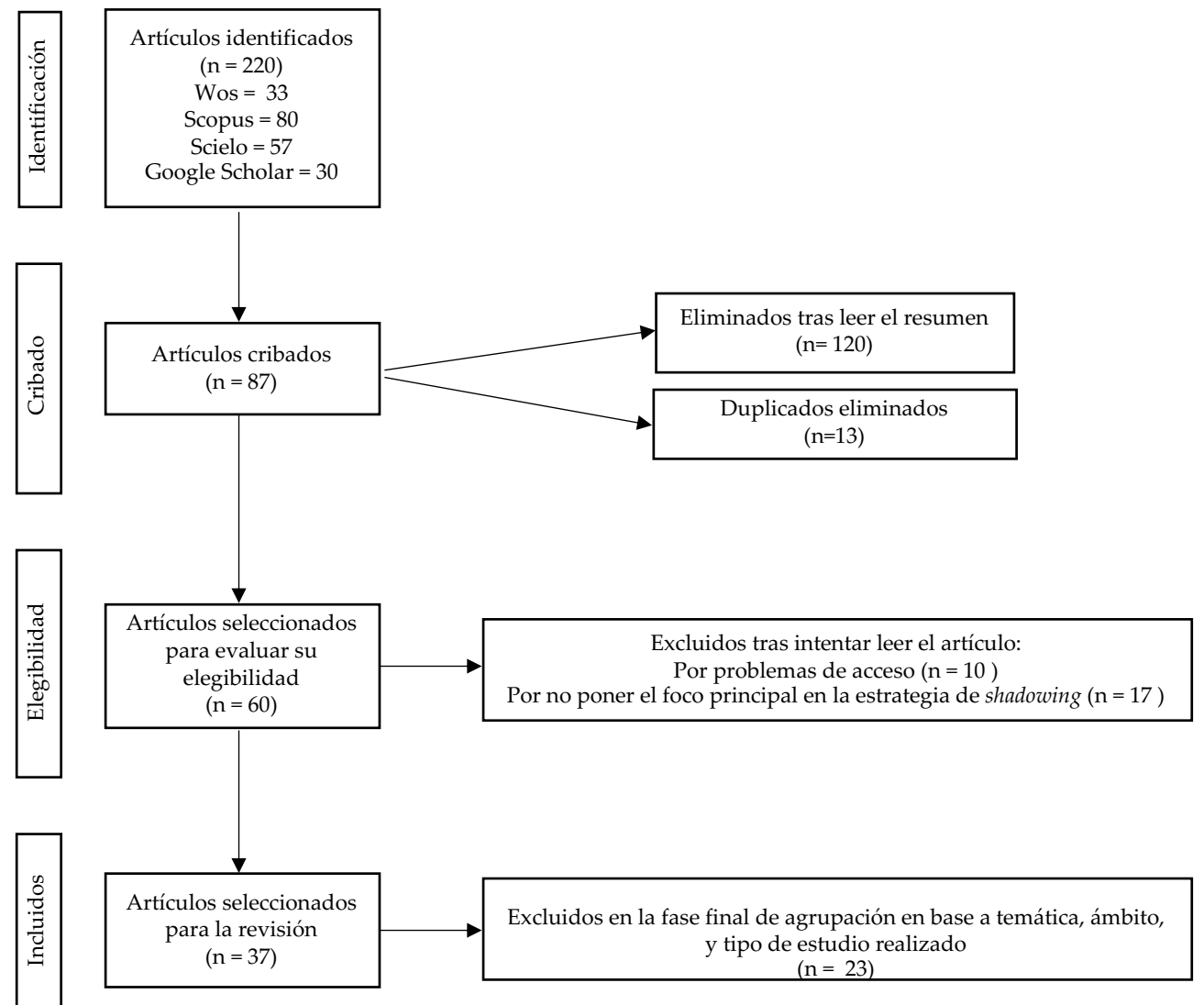

Figura 1. Diagrama de flujo según PRISMA.

Estos 37 artículos fueron agrupados en base al tipo de estudio realizado, vale decir: descriptivos, valorativos o empíricos. Una vez hecha esta agrupación, se llevó a cabo una codificación de tipo axial (Strauss y Corbin, 2016) a través de redes de códigos que terminaron por generar tres categorías emergentes donde se agrupó el contenido de los artículos: (1) Origen, ámbitos de aplicación y caracterización; (2) Experiencias y usos; (3) Implicaciones ético-pragmáticas.

\section{Resultados}

La síntesis de los resultados se muestra en la Tabla 1, estructurada de forma cronológica y alfabética. 
Tabla 1.

Síntesis de artículos seleccionados

\begin{tabular}{|c|c|c|c|c|}
\hline $\mathbf{N}^{\circ}$ & Autores & Año & Publicación & $\begin{array}{l}\text { Tipo de estudio } \\
\text { realizado }\end{array}$ \\
\hline 1 & Alaimo, A. y Picone, M. & 2015 & Scienze Del Territorio. & Descriptivo \\
\hline 2 & $\begin{array}{l}\text { Alirezabeigi, S., Masschelein, } \\
\text { J., y Decuypere, M. }\end{array}$ & 2020 & $\begin{array}{l}\text { Learning, Media and Tech- } \\
\text { nology. }\end{array}$ & Empírico \\
\hline 3 & Bartkowiak-Théron, I. & 2013 & Social Research Methods. & Descriptivo \\
\hline 4 & $\begin{array}{l}\text { Bartkowiak-Theron, I., y Robyn } \\
\text { Sappey, J. }\end{array}$ & 2012 & Qualitative Research Journal. & Descriptivo \\
\hline 5 & Bethune, J. y Gilbert, J. & 2019 & $\begin{array}{l}\text { Oxford Research Encyclope- } \\
\text { dias. }\end{array}$ & Valorativo \\
\hline 6 & Blake, K., y Stalberg, E. & 2009 & Serials Review. & Descriptivo \\
\hline 7 & $\begin{array}{l}\text { Bøe, M., Hognestad, K., y Wan- } \\
\text { iganayake, M. }\end{array}$ & 2017 & $\begin{array}{l}\text { Educational Management } \\
\text { Administration \& } \text { Leader- } \\
\text { ship. }\end{array}$ & Empírico \\
\hline 8 & Bueger, C., y Gadinger, F. & 2018 & International Practice Theory. & Empírico \\
\hline 9 & Carrasco, J., y Medina, S. & 2019 & $\begin{array}{l}\text { Physis: Revista de Saúde Co- } \\
\text { letiva. }\end{array}$ & Empírico \\
\hline 10 & $\begin{array}{l}\text { Coker, E., Ploeg, J., Kaasalain- } \\
\text { en, S., y Carter, N. } \\
\end{array}$ & 2017 & \begin{tabular}{|l|}
$\begin{array}{l}\text { International Journal of Old- } \\
\text { er People Nursing. }\end{array}$ \\
\end{tabular} & Empírico \\
\hline 11 & Czarniawska, B. & $2014 b$ & $\begin{array}{l}\text { Qualitative Research in Orga- } \\
\text { nizations and Management: } \\
\text { An International Journal. }\end{array}$ & Empírico \\
\hline 12 & Czarniawska, B. & $2018 b$ & $\begin{array}{l}\text { Qualitative Methodologies in } \\
\text { Organization Studies. }\end{array}$ & Descriptivo \\
\hline 13 & Ferguson, K. & 2016 & Reflective Practice. & Descriptivo \\
\hline 14 & Gill, R. & 2011 & $\begin{array}{l}\text { Qualitative Research in Orga- } \\
\text { nizations and Management: } \\
\text { An International Journal. }\end{array}$ & Empírico \\
\hline 15 & Gill, R., Barbour, J., y Dean, M. & 2014 & \begin{tabular}{|l|} 
Qualitative Research in Orga- \\
nizations and Management: \\
An International Journal. \\
\end{tabular} & Valorativo \\
\hline 16 & Gobo, G. & 2005 & $\begin{array}{l}\text { Forum Qualitative Sozial } \\
\text { forschung / Forum: Qualita- } \\
\text { tive Social Research. }\end{array}$ & Descriptivo \\
\hline 17 & Gorup, M. & 2016 & Emerald. & Valorativo \\
\hline 18 & Hirsh, S. G. & 1999 & $\begin{array}{l}\text { Journal of The American So- } \\
\text { ciety for Information Science. }\end{array}$ & Empírico \\
\hline 19 & Hognestad, K., y Bøe, M. & 2016 & \begin{tabular}{|l|} 
European Early Childhood \\
Education Research Journal. \\
\end{tabular} & Empírico \\
\hline 20 & Jirón, P. & 2012 & Bifurcaciones. & Empírico \\
\hline
\end{tabular}




\begin{tabular}{|c|c|c|c|c|}
\hline $\mathbf{N}^{\circ}$ & Autores & Año & Publicación & $\begin{array}{l}\text { Tipo de estudio } \\
\text { realizado }\end{array}$ \\
\hline 21 & Johnson, B. & 2014 & $\begin{array}{l}\text { Qualitative Research in Orga- } \\
\text { nizations and Management: } \\
\text { An International Journal. }\end{array}$ & Valorativo \\
\hline 22 & Kum, P. & 2017 & $\begin{array}{l}\text { Journal of Historical Archae- } \\
\text { ology \& Anthropological Sci- } \\
\text { ences }\end{array}$ & Empírico \\
\hline 23 & Knutas, A. & 2019 & Qualitative Inquiry & Empírico \\
\hline 24 & Kusnoor y Stelljes, 2016 & 2016 & Medical Teacher. & Valorativo \\
\hline 25 & $\begin{array}{l}\text { Lindberg, K., y Czarniawska, } \\
\text { B. }\end{array}$ & 2006 & $\begin{array}{|lll|}\text { Scandinavian } & \text { Journal } \\
\text { Managemen. }\end{array}$ & Descriptivo \\
\hline 26 & McDonald, S. & 2005 & Qualitative Research. & Descriptivo \\
\hline 27 & McDonald, S., y Simpson, B. & 2014 & $\begin{array}{l}\text { Qualitative Research in Orga- } \\
\text { nizations and Management: } \\
\text { An International Journal. } \\
\end{array}$ & Descriptivo \\
\hline 28 & Miller, P. & 1998 & $\begin{array}{|lll|}\begin{array}{l}\text { European } \\
\text { view. }\end{array} & \text { Accounting Re- } \\
\end{array}$ & Valorativo \\
\hline 29 & Mintzberg, H. & 1970 & $\begin{array}{|ll|}\begin{array}{l}\text { Administrative } \\
\text { Quarterly. }\end{array} & \text { Science } \\
\end{array}$ & Descriptivo \\
\hline 30 & Padgett, D. & 2017 & Sage. & Descriptivo \\
\hline 31 & Quinlan, E. & 2008 & Qualitative Inquiry. & Descriptivo \\
\hline 32 & Schuler, M. & 2016 & Nurse Educator. & Empírico \\
\hline 33 & Sisto, V., y Zelaya, V. & 2013 & Universitas Psychologica. & Empírico \\
\hline 34 & Tengblad, S. & 2003 & $\begin{array}{|lll|}\text { Scandinavian Journal of } \\
\text { Management. }\end{array}$ & Empírico \\
\hline 35 & Tengblad, S. & 2006 & $\begin{array}{|ll|}\begin{array}{l}\text { Journal of Management } \\
\text { Studies }\end{array} & \\
\end{array}$ & Empírico \\
\hline 36 & $\begin{array}{l}\text { Vuorikari, R., y Castaño-Muñoz, } \\
\text { J. }\end{array}$ & 2016 & $\begin{array}{l}\text { Joint Research Centre Science for } \\
\text { Policy Report (EC) }\end{array}$ & Valorativo \\
\hline 37 & Wolcott, H. & 2014 & $\begin{array}{l}\text { Qualitative Research in Orga- } \\
\text { nizations and Management: } \\
\text { An International Journal. }\end{array}$ & Descriptivo \\
\hline
\end{tabular}

A continuación, se exponen los hallazgos agrupados en las tres categorías antes mencionadas.

\section{Origen, ámbitos de aplicación y caracterización de la estrategia}

El origen del shadowing es múltiple y puede ser rastreado paralelamente en diversas disciplinas y campos de estudio en ciencias sociales (Czarniawska, 2014a; 2018; Gobo, 2005; McDonald, 2005Mintzberg, 1970; Alaimo y Picone, 2015). En el ámbito de los estudios industriales, organizacionales y de gestión (Bartkowiak-Theron y Robyn, 2012; 
Johnson, 2014; Lindberg y Czarniawska, 2006; Czarniawska, 2018b); en el ámbito del trabajo social (Padgett, 2017), en estudios de información (Hirsh, 1999), en sociología del consumo (Miller, 1998); en ciencias de la salud (Coker et al., 2016; McDonald, 2005; Kusnoor y Stelljes, 2016; Schuler, 2016); y finalmente en ciencias de la educación (Gorup, 2016; Hognestad y Bøe, 2016; Vuorikari y Castaño-Muñoz, 2016).

En coherencia con lo señalado, las unidades objeto/sujeto de estudio mediante esta estrategia son igualmente variadas; tal como lo evidencian los trabajos revisados, el shadowing ha sido aplicado a objetos, sujetos o prácticas. Por ejemplo, Carrasco y Medina (2019) y Sisto y Zelaya (2013), sombrean objetos, programas informáticos e instrumentos de evaluación respectivamente. Coker et al (2016) prácticas de higiene oral en hospitales, mientras que Schuler (2016) las transformaciones actitudinales de estudiantes de enfermería.

Si bien no todos los autores y autoras hacen referencia explícita al shadowing en sus trabajos (Gorup, 2016), la mayoría comparte la preocupación de modificar las formas tradicionales de observación a partir de argumentos tales como: la intensión de registrar la movilidad, el aspecto procedural, y la constante transformación de los fenómenos contemporáneos (McDonald, 2005; Mintzberg; 1970; Miller, 1998). Este panorama ilustra la pluralidad de disciplinas y áreas de estudios donde resuenan las transformaciones de observación en el campo.

Con relación a su caracterización, el shadowing es definido unánimemente como un método cualitativo, donde el/la investigador/a sigue (o sombrea) por un período de tiempo una persona, una actividad $\mathrm{u}$ objeto con el interés de captar a través de la observación en movimiento, todo aquello que sucede en el flujo diario de la unidad observada (Gill et al., 2014; Gill, 2011; Carrasco y Medina, 2019). Pese a este consenso, identificamos diferentes tipos de shadowing, diferencias dadas principalmente por el propósito de quien sombrea (Mcdonald y Simpson, 2014). En este sentido, el shadowing no siempre es puesto en marcha como una estrategia de investigación ya que no siempre tiene el propósito de generar conocimiento. Por ejemplo, hay casos donde la estrategia ha sido utilizada en formación o actualización profesional, o para realizar diagnósticos institucionales orientados a la resolución de problemas (Gorup, 2016; Bøe et al., 2016). Profundizaremos en la caracterización a través de tres subcategorías claves: 1) la noción de seguimiento; 2) la actitud de extrañeza; y 3) la distancia con la observación tradicional.

\section{La noción de seguimiento}

Se asocia al ejercicio del/a investigador/a de seguir la unidad de estudio (individual, grupal, humana o no-humana) por el campo investigado provocando el registro de un flujo continuo de datos (Alirezabeigi et al., 2020; Schuler, 2016). Los trabajos revisados muestran que con el registro del flujo de la unidad se busca dar cuenta de la complejidad del fenómeno investigado, registrando polifonías, interacciones y acciones en un contexto delimitado (Bartkowiak-Therón, 2013; McDonald, 2005).

\section{La actitud de extrañeza}

En los trabajos revisados se tiende a describir la "sombra" (el/a investigador/a) como un actor que puede ver cosas diferentes a los actores nativos, precisamente 
porque este contexto le es ajeno (Kum, 2017; Knutas, 2019). Se trata de la experiencia de extrañeza como actitud fundamental para visibilizar detalles cotidianos que pueden pasar inadvertidos para la comunidad estudiada. Pese a la pluralidad de usos de esta técnica, una característica transversal es usar la posición de extranjero para reconocer las diferencias y buscar la comprensión de la unidad estudiada (Knutas, 2019). De hecho, Czarniawska (2018b) advierte como la actitud de extrañeza aumenta -no disminuye como podría pensarse desde un sentido común antropológico- en la medida que aumenta a su vez el grado comprensión de la unidad de estudio.

\section{Las diferencias con la observación tradicional}

Es frecuente comparar el shadowing con las técnicas clásicas de observación directa y/o participante y distinguirlas mediante 2 elementos. Primero, la idea de flujo como organizador de los datos a registrar; y segundo, que el registro no se limita a lo observado sino además a los reportes verbales elaborados por los actores (Bethune, y Gilbert, 2019; Czarniawska, 2014b, 2018b; Quinlan, 2008; McDonald y Simpson, 2014). Por ejemplo, el trabajo de Alirezabeigi Masschelein y Decuypere (2020), aborda los incidentes críticos que ocurren en escuelas que promueven el uso escolar de ordenadores personales desde un enfoque etnográfico sociomaterial, poniendo el acento en la constante interacción-creación de cosas, tiempos y espacios escolares.

\section{Experiencias y usos de la estrategia}

Los resultados se agrupan en 2 subcategorías: argumentos para seleccionar la técnica y procedimiento de utilización.

\section{Argumentos para seleccionar la técnica}

Una de las razones más frecuentes para seleccionar esta estrategia es su capacidad única para proporcionar información sobre el día a día de las actividades en un contexto específico (McDonald, 2005; Mintzberg, 1970; Wolcott, 2014). Esto se articula con lo señalado con McDonald y Simpson (2014) cuando explican que el sombreamiento explora prácticas sin desconectar su registro de la dimensión espacio-temporal en que se producen, aspecto que otras técnicas por sus limitaciones internas, no serían capaces de explorar. Por ejemplo, se accede a situaciones que brindan información valiosa y que habitualmente quedan fuera del alcance (llamadas telefónicas, conversaciones espontáneas o solicitudes inesperadas), las cuales transcurren mientras la sombra observa. Esta centralidad del movimiento explica que el shadowing sea una técnica física, mental y emocionalmente exigente, y, por tanto, desafiante en términos investigativos (Ferguson, 2016).

La estrategia suele ser utilizada ante la necesidad de una investigación que requiere datos de una cualidad diferente a lo textual o visual. Usualmente son estudios empíricos que buscan responden a preguntas de investigación relativa al ¿Cómo?, indagando en qué modo se desarrollan diferentes actividades y como éstas son explicadas por los y las participantes (Czarniawska, 2014b; Tengblad, 2003; 2006). En base a su trabajo en 
educación superior, por ejemplo, Gorup (2016) plantea que el sombreamiento permite ir más allá de la descripción general de los comportamientos previstos o prescritos, estudiando actividades y percepciones de ciertos/as sujetos/as a medida que ocurren. Esto permite, como señalamos en el apartado anterior, acceder a la dimensión no documentada de los procesos formales.

\section{¿Cómo se usa el shadowing?}

El sombreamiento se presenta ante todo como estrategia de naturaleza abierta y flexible. Esta característica según Gill et al. (2014), requiere que investigadores e investigadoras deban tomar permanentemente decisiones durante el proceso. La flexibilidad de la estrategia explica por tanto la variabilidad en cada experiencia investigativa; no hay una manera unívoca de implementación, sino que se va ajustando a las necesidades que la propia realidad estudiada exija. Pese a ello, nuestros análisis evidencian de todas formas ciertas regularidades. La primera, tiene que ver con definir y delimitar aquello que se va a sombrear, en definir una unidad de seguimiento. El shadowing puede centrarse en una o varias personas de un determinado grupo; la unidad de observación o seguimiento puede ser también una acción desarrollada por diferentes personas. En segundo lugar, la duración del seguimiento. Los estudios analizados reportan trabajos de campo de un solo día, un mes o incluso años (Coker et. al, 2016; Wolcott, 2014). Así mismo, los períodos de observación pueden ser consecutivos o no. De todas formas, lo que se evidencia es que la duración del uso de la técnica guarda relación directa con la pregunta de investigación y de las cualidades de la unidad que se está estudiando (Johnson, 2014). Un tercer punto refiere al formato de registro del trabajo de campo. Hay consenso en que el investigador o investigadora registrará una serie casi continua de notas escritas o audio y aquello registrado varía en directa relación con la cantidad y calidad de las unidades observadas (Knutas, 2019; Kum, 2017). Se puede registrar sobre participantes, objetos, acciones, interacciones, tiempos y contenidos de conversaciones, así como respuestas a las preguntas formuladas.

Ahora bien, uno de los puntos de mayor divergencia refiere a las preguntas que hace el/la investigador/a. De acuerdo con nuestros análisis, algunas preguntas son de carácter aclaratorio como en el estudio de Kusnoor y Stelljes (2016) con estudiantes de medicina preclínica; otras de naturaleza argumental como el caso del enfoque paxiográfico de Bueger y Gadinger (2018), quienes proponen entrevistas conversacionales con los y las participantes para recrear sus prácticas. En algunos casos, sin embargo, las preguntas no están predefinidas. El trabajo de Bøe et al (2016) incorpora encuentros preliminares con los centros de educación primaria para responder las dudas de los participantes, previo al inicio del sombreamiento.

\section{Implicaciones ético-pragmáticas}

Lo relativo a las implicancias ético-pragmáticas hace referencia a los aspectos valorativos del shadowing por parte de usuarios y usuarias. Este apartado se compone de 
dos subcategorías: la relación investigador/a-investigado/a y la cualidad del dato en la comprensión profunda del shadowing ${ }^{6}$.

\section{Relación investigador/a-investigado/a}

Entre los aspectos evaluados positivamente en la literatura, es frecuente el trabajo colaborativo como estilo relacional que permite una cuidadosa coordinación entre investigador/a e investigado/a, lo que redunda en un beneficio de la actividad de ambos. Esto ya que, por un lado, aumenta la capacidad de comprensión de la realidad estudiada, y por otro, supone una experiencia de reflexión para el propio agente sombreado (Gillet al., 2014; Jirón, 2012; Czarniawska, 2018b). De esta forma, el shadowing es una técnica colaborativa en la medida en que sombra y sombreado interactúan, representan roles, crean conocimiento, a la vez que generan comprensión de lo performado (Gill, 2011; Vuorikari y Castaño-Muñoz, 2016), favoreciendo un mayor compromiso con el trabajo investigativo y colaboración con los objetivos de la investigación (Bøe et al., 2016; Gill et al., 2014; Jirón, 2012). El sombreado es además un enfoque que asume que los y las participantes son expertos/as en sus propias prácticas (Bueger y Gadinger, 2018), y que incluso pueden llegar a constituirse en un espacio de aprendizaje para sus participantes, incluido el equipo investigador.

Pero este mismo aspecto se ve cuestionado en algunos textos cuando se refieren a la relación de la sombra con quienes son investigados. Algunos autores reportan que los sujetos sombreados se han sentido evaluados (Gill et al., 2014), invadidos (Johnson, 2014) o incomodados por la exposición que supone la técnica (Mintzberg, 1970).

Algunos autores advierten del potencial riesgo de transformarse en nativos o nativas y perder así la distancia en la observación. Este riesgo está presente especialmente en aquellas investigaciones de larga duración y/o presencia en el campo, lo que puede conllevar a perder de vista los objetivos de la investigación, así como también una actitud de normalización y/o naturalización de los hechos observados.

\section{La cualidad del dato en la comprensión profunda del shadowing}

En lo referido a la cualidad de los datos, éstos adquieren mayor densidad que aquellos recopilados a través de otras técnicas clásicas (observación, entrevista), puesto que en el shadowing son producidos en su contexto natural incorporando frecuentemente datos observacionales y datos conversacionales, con toda la riqueza que esto supone. En esa línea, Blake y Stalberg (2009) y McDonald (2005) señalan que este tipo de datos permite una comprensión holística, acceso a capas profundas de lo estudiado, así como el trabajo con unidades de análisis más complejas e interrelacionadas. El sombreamiento ubica a fin de cuentas al investigador o investigadora en una posición privilegiada que le permite experienciar junto a los y las participantes las prácticas que se están sombreando (Hognestad y Bøe, 2016; Knutas, 2019). Esto permite a los investigadores

6 Asumiendo que en toda estrategia de investigación existen elementos positivos y negativos que influyen tanto en observadores, participantes e investigadores, nos centraremos en aquellas precauciones que convendría tener en cuenta con relación a estos aspectos valorativos, al momento de emplear el shadowing en una tarea de investigación. 
e investigadoras "ponerse en los zapatos" de los participantes, así como participar de sus prácticas, lo que otorga una posibilidad de acceder a niveles de profundidad indiscutiblemente de mayor riqueza que técnicas clásicas (Ferguson, 2016).

\section{Discusión y conclusiones}

Nuestros resultados evidencian que el shadowing no ha sido considerado aún un objeto de estudio propio, con una adecuada discusión respecto a sus dimensiones epistemológicas o metodológicas, y pese a que ha sido tratado predominantemente como una técnica (más) de producción de datos y no como una estrategia de investigación, se evidencia el promisorio rol que esta técnica podría desempeñar de cara a una nueva investigación educativa. Tomando como punto de partida la caracterización sobre el shadowing -sus principales ámbitos y formatos de implementación, así como implicancias y usos-, el trabajo de revisión y sistematización realizado, proponemos 4 ejes de de contribución para la investigación educativa; del más específico al más global éstos son: i) la incorporación de nuevas dimensiones de las unidades estudiadas; ii) la inclusión de participantes/saberes tradicionalmente excluidos; iii) el desarrollo de niveles de profundidad comprensiva más sofisticados y; iv) la potenciación de una mayor autonomía disciplinar en la investigación educativa.

\section{La incorporación de nuevas dimensiones de las unidades de estudio}

En directa relación con el predominio de las etnografías relámpago, el shadowing permite incorporar elementos que tradicionalmente han sido postergados en la investigación educativa: la arquitectura e infraestructura escolar como dimensiones que componen el fenómeno educativo (la disposición espacial de las aula, los recursos pedagógicos); las dimensiones corporales y emocionales de la experiencia educativa (la afectividad del trabajo docente, el vínculo docente-estudiante); la materialidad y el rol de los actores educativos no-humanos en la configuración de la experiencia escolar (libros de clases, instrumentos de gestión, orientaciones gubernamentales). Todos estos elementos, decisivos en la configuración de la cultura y la cotidianeidad escolar, podrían ser (re) incorporados en la comprensión de los fenómenos educativos a partir de la implementación del shadowing. Esto permitiría a su vez, contribuir al propio desarrollo del shadowing como perspectiva de investigación, toda vez que la etnografía es a fin de cuentas un trabajo artesanal (Denzin y Lincoln, 2018), un oficio que se crea y recrea a partir de su implementación y reflexión del propio uso.

\section{La inclusión de participantes/saberes tradicionalmente excluidos}

El shadowing posibilita incorporar actores tradicionalmente catalogados como "no expertos" y por tanto históricamente marginados de los procesos investigativos. Sostenemos que la posibilidad de incorporar a actores no expertos no se reduce a una iniciativa metodológica valorable, sino que es condición necesaria para un riguroso despliegue de la estrategia. En efecto, el diseño y desarrollo del shadowing sólo es posible en un contexto de alianza y colaboración entre la comunidad escolar y el equipo investigador. 
Esto permite que el shadowing se constituya en una valiosa oportunidad metodológica -y ética- de trascender la naturaleza extractiva de la investigación educativa actual (Bøe et al., 2016; Bueger y Gadinger, 2018; Biesta, 2015), avanzando hacia nuevas lógicas y formatos que reconozcan, legitimen e incorporen en un lugar protagónico los y las participantes de investigaciones y sus respectivos saberes. El shadowing facilita a fin de cuentas avanzar hacia una nueva ecología de los saberes en la investigación educativa.

\section{Desarrollar niveles de comprensión más sofisticados}

El shadowing resulta un aporte evidente en investigaciones que busquen una comprensión profunda de sus fenómenos de estudio. Como advierte Hammerley (2018), gran parte de la etnografía escolar actual, por ejemplo, no sólo ha confundido etnografía con trabajo de campo haciéndolos términos equivalentes, sino además ha privilegiado el formato de etnografía relámpago, que sólo posibilita componer retratos superficiales de lo estudiado. Un aspecto distintivo del shadowing es la cualidad de sus datos, aportando unas descripciones densas y captando estructuras de significación profundas de los fenómenos educativos (Gorup, 2016). Con todo, no solo se trata de generar datos y análisis complejos, sino también densamente conectados con sus contextos de producción, la cultura escolar de las propias comunidades escolares, por ejemplo, así como los entornos socioculturales en que éstas se encuentran emplazadas. Por esta razón el shadowing educativo podría posicionarse como una estrategia pertinente para una investigación educativa orientada desde y para las necesidades situadas del contexto escolar que actúe como telón de fondo de la investigación etnográfica.

\section{Potenciar una mayor autonomía disciplinar de la investigación educativa}

Aplicando el shadowing a la investigación educativa, el o la docente o miembro de la comunidad educativa que lo utiliza se convierte en investigador cuyo proceder está acreditado y justificado a nivel de validez y garantías científicas cualitativas por el procedimiento del shadowing, siempre que este se utilice correctamente. Podríamos concluir que el shadowing es una adaptación del procedimiento etnográfico tradicional a la época actual en la que ni el investigador ni los participantes tienen el suficiente tiempo y los recursos ideales para adentrarnos ilimitadamente en su quehacer cotidiano. Esto, focalizado al ámbito de la investigación educativa, permite a docentes y al resto de la comunidad educativa obtener información, reflexionar, analizar y transformar la realidad en la que están inmersos diariamente.

Finalmente, sostenemos que la investigación educativa necesita de métodos, estrategias y herramientas que permitan a esta esfera del conocimiento emanciparse del conocimiento psicológico, sociológico o managerial -entre otros- y desarrollar sus propios métodos, formatos y saberes a múltiple nivel: desde lo estrictamente filosóficoepistemológico, hasta lo más práctico y situado en el propio quehacer y devenir de la escuela y de los distintos actores implicados en la misma. Frente a este desafío, como argumenta este trabajo, el shadowing puede ser de gran utilidad para profundizar en las necesidades que el campo escolar necesita a la luz de las actuales transformaciones 
socioculturales que atraviesan los contextos educativos en nuestras sociedades contemporáneas, como ha sido, por ejemplo, la educación a distancia en tiempos de pandemia.

\section{Bibliografía}

Alaimo, A. \& Picone, M. (2015). Shadowing and qualitative Gis: Tools for Urban Narrations. Scienze del territorio, 3, 391-400.

Alirezabeigi, S., Masschelein, J., \& Decuypere, M. (2020). Investigating digital doings through breakdowns: A sociomaterial ethnography of a Bring Your Own Device school. Learning, Media and Technology, 45(2), 193-207. https://doi.org/10.1080/1743 9884.2020.1727501

Baleriola, E. (2018). Observación Sindrómica. Gestión de la Vida en el Siglo XXI. Athenea Digital, 18(3), e-2325. http://dx.doi.org/10.5565/rev/athenea.2325

Bartkowiak-Théron, I. (2013). Social Research Methods 3rd edition. Oxford University.

Bartkowiak-Theron, I. \& Robyn, J. (2012). The Methodological Identity of Shadowing in Social Science Research. Qualitative research journal, 12(1), 7-16. https://doi. org/10.1108/14439881211222697

Beach, D., Bagley, C., \& Marques, S. (2018). The Wiley handbook of ethnography of education. John Wiley \& Sons.

Bethune, J. \& Gilbert, J. (2019). School ethnography: Critical questions and methods. Oxford Research Encyclopedia of Education. https://doi.org/10.1093/acrefore/9780190264093.013.338

Biesta, G. (2015). Good education in an age of measurement: Ethics, politics, democracy. Paradigm Publishers.

Blake, K. \& Stalberg, E. (2009). Me and my shadow: Observation, documentation, and analysis of serials and electronic resources workflow. Serials Review, 35(4), 242-252. https://doi.org/10.1016/j.serrev.2009.08.018

Bøe, M., Hognestad, K. \& Waniganayake, M. (2016). Qualitative shadowing as a research methodology for exploring early childhood leadership in practice. Educational Management Administration \& Leadership, 45(4), 605-620. https://doi. org/10.1177\%2F1741143216636116

Bru Luna, L. M., Martí-Vilar, M. \& González-Sala, F. (2020). Revisión sistemática de intervenciones en prosocialidad y empatía en personas con TEA. Revista de Investigación Educativa, 38(2), 359-377. http://dx.doi.org/10.6018/rie.395421

Bueger, C., \& Gadinger, F. (2018). Doing Praxiography: Research Strategies, Methods and Techniques. In C. Bueger \& F. Gadiner (eds.), International Practice Theory, (pp. 131-161). Palgrave Macmillan. https://doi.org/10.1007/978-3-319-73350-0_6

Carrasco, J. \& Medina, S. (2019). El Sistema Informático de la Reforma GES en Chile: una etnografía de dispositivos de gobierno sanitario. Physis: Revista de Saúde Coletiva, 28(4), 1-21. http://dx.doi.org/10.1590/s0103-73312018280424

Coker, E., Ploeg, J., Kaasalainen, S., \& Carter, N. (2017). Nurses' oral hygiene care practices with hospitalised older adults in postacute settings. International Journal of Older People Nursing, 12(1), e12124. https://doi.org/10.1111/opn.12124 
Contreras, P., Assael, J., Acuña, F., Santa Cruz, E., Campillay, B., \& Pujadas, B. (2016). Building ethnographic knowledge: Reflections from field experience in school institutions. Athenea Digital, 16(3), 55-79. https://doi.org/10.5565/rev/athenea.1629

Cooper, C., Booth, A., Varley-Campbell, J., Britten, N., \& Garside, R. (2018). Defining the process to literature searching in systematic reviews: a literature review of guidance and supporting studies. BMC Medical Research Methodology, 18(1), 1-14. https://doi.org/10.1186/s12874-018-0545-3

Czarniawska, B. (2014a). Social Science Research: From Field to Desk. SAGE Publications.

Czarniawska, B. (2014b). Why I think shadowing is the best field technique in management and organization studies. Qualitative Research in Organizations and Management, 9(1). 90-93. https://doi.org/10.1108/QROM-02-2014-1198

Czarniawska, B. (2018a). Organizing: How to study it and how to write about it. Qualitative Research in Organizations and Management: An International Journal, 3(1), 4-20. https://doi.org/10.1108/17465640810870364

Czarniawska, B. (2018b). Fieldwork Techniques for Our Times: shadowing. In M. Ciesielska \& D. Jemielniak. (Eds.), Qualitative Methodologies in Organization Studies (pp.53-74). Palgrave Macmillan. https://doi.org/10.1007/978-3-319-65442-3_3

Denzin, N. K. \& Lincoln, Y. S. (2018). The SAGE Handbook of Qualitative Research. SAGE.

Delamont, S. \& Atkinson, P. (2018). The ethics of ethnography. In R. Iphofen, \& M. Tolich. The Sage Handbook of Qualitative Research Ethics (pp. 119-132). SAGE. https:// doi.org/10.4135/9781526435446

Earley, P. (2012). Observation Methods: Learning about Leadership Practice through shadowing. Educational, Cultural and Psychological Studies, 6, 15-31. https://doi. org/10.7358/ecps-2012-006-earl

Engeström, Y. \& Sannino, A. (2016). El aprendizaje expansivo en movimiento: aportaciones de la investigación en curso, Infancia y Aprendizaje, 39(3), 401-435.

Erstad, O., Miño, R., \& Rivera-Vargas, P. (2021). Prácticas educativas para transformar y conectar escuelas y comunidades. Comunicar: Revista Científica de Comunicación y Educación, 29(66). 21-31. http://dx.doi.org/10.3916/C66-2021-01

Fardella, C. (2020). Abrir la jaula de oro. La Universidad Managerial y Sus Sujetos. Izquierdas, (49), 2299-2320. http://www.izquierdas.cl/images/pdf/2020/n49/ art110_2299_2320.pdf

Farley-Ripple, E., May, H., Karpyn, A., Tilley, K., \& McDonough, K. (2018). Rethinking Connections Between Research and Practice in Education: A Conceptual Framework. Educational Researcher, 47(4), 235-245. https://doi.org/10.3102/0013189X18761042

Ferguson, K. (2016). Lessons learned from using shadowing as a qualitative research technique in education. Reflective Practice, 17(1), 15-26, https://doi.org/10.1080/146 23943.2015.1123683

Gill, R. (2011). The shadow in organizational ethnography: moving beyond shadowing to spect-acting. Qualitative Research in Organizations and Management, 6(2), 155-133. https://doi.org/10.1108/17465641111159116

Gill, R, Barbour, J., \& Dean, M. (2014). Shadowing in/as work: ten recommendations for shadowing fieldwork practice. Qualitative Research in Organizations and Management, 9(1), 69-89. https://doi.org/10.1108/QROM-09-2012-1100 
Gobo, G. (2005). The renaissance of qualitative methods. Forum: Qualitative Social Research, 6(3). 1-12. http://dx.doi.org/10.17169/fqs-6.3.5

Gorup, M. (2016). Studying higher education close-up: unexplored potentials of" shadowing" in higher education research. In J. Huisman \& M. Tight (Eds.), Theory and method in higher education research (pp. 135-15). Emerald. https://doi.org/10.1108/ S2056-375220160000002007

Hammersley, M. (2018) What is ethnography? Can it survive? Should it? Ethnography and Education, 13(1), 1-17. https://doi.org/10.1080/17457823.2017.1298458

Hammersley, M. \& Atkinson, P. (2005). Etnografía. Métodos de investigación. Paidós.

Hirsh, S. (1999). Children's relevance criteria and information seeking on electronic resources. Journal of the American Society for information Science, 50(14), 1265-1283. https://doi.org/10.1002/(SICI)1097-4571(1999)50:14\%3C1265::AID-ASI2\%3E3.0.CO;2-E

Hognestad, K. \& Bøe, M. (2016). Studying practices of leading-Qualitative shadowing in early childhood research. European Early Childhood Education Research Journal, 24(4), 592-601. https://doi.org/10.1080/1350293X.2016.1189725

Jiménez, F., Fardella, C. \& Muñoz-Proto, C. (2017). Una aproximación microetnográfica de prácticas pedagógicas en escuelas multiculturales. tensiones y desafíos en torno a la escolarización de inmigrantes y grupos minoritarios. Perfiles Educativos, 39(156), 72-88.

Jirón, P. (2012). Transformándome en la sombra. Bifurcaciones, 10, 1-14. http://www. bifurcaciones.cl/2012/11/transformandome-en-la-sombra/

Johnson, B. (2014). Ethical issues in shadowing research. Qualitative Research in Organizations and Management, 9(1), 21-40. https://doi.org/10.1108/QROM-09-2012-1099

Kusnoor, A. V., \& Stelljes, L. (2016). Interprofessional learning through shadowing: Insights and lessons learned. Medical Teacher, 38(12), 1278-1284. https://doi.org/10. 1080/0142159X.2016.1230186

Kum, P. (2017). Tackling strangeness while conducting ethnographic fieldwork by an anthropologist in Africa: a narrative from Cameroon, Journal of Historical Archaeology $\mathcal{E}$ Anthropological Sciences, 1(5), 172-180. https://doi.org/10.15406/jhaas.2017.01.00028

Knutas, A. (2019). Shadowing or What? Experience of shadowing Acts of Being in the Field of Education. Qualitative Inquiry, 25(7), 661-669. https://doi. org/10.1177/1077800418806620

Lindberg, K. \& Czarniawska, B. (2006). Knotting the action net, or organizing between organizations. Scandinavian Journal of Management, 22(4), 292-306. https://doi. org/10.1016/j.scaman.2006.09.001

McDonald, S. (2005). Studying actions in context: a qualitative shadowing method for organizational research. Qualitative Research, 5(4), 455-473. https:/doi. org/10.1177\%2F1468794105056923

McDonald, S. \& Simpson, B. (2014). Shadowing research in organizations: the methodological debates. Qualitative Research in Organizations and Management, 9(1), 3-20. https://doi.org/10.1108/QROM-02-2014-1204

Miller, P. (1998). The Margins of Accounting. European Accounting Review, 7(4), 605-621. https://doi.org/10.1111\%2Fj.1467-954X.1998.tb03474.x 
Mintzberg, H. (1970). Structured observation as a method to study managerial work. Journal of management studies, 7(1), 87-104. https://doi.org/10.1111/j.1467-6486.1970. tb00484.x

Padgett, D. (2017). Qualitative Methods in Social Work Research. SAGE.

Quinlan, E. (2008). Conspicuous Invisibility: Shadowing as a Data Collection Strategy. Qualitative Inquiry, 14(8), 1480-1499. https://doi.org/10.1177/1077800408318318

Sancho-Gil, J. M., Rivera-Vargas, P., \& Miño-Puigcercós, R. (2020). Moving beyond the predictable failure of Ed-Tech initiatives. Learning, Media and Technology, 45(1), 61-75. http://dx.doi.org/10.1080/17439884.2019.1666873

Schuler, M. (2016). Shadowing in early baccalaureate nursing education and its influence on professional role perspectives. Nurse Educator, 41(6), 304-308. https:// doi.org/10.1097/NNE.0000000000000276

Sisto, V., \& Zelaya, V. (2013). La Etnografía de Dispositivos y el Estudio de los Instrumentos de Rendición de Cuentas como Prácticas. Universitas Psychologica, 12(4), 1345-1354. https://doi.org/10.11144/Javeriana.UPSY12-4.edha

Strauss, A. \& Corbin, J. (2016). Bases de la investigación cualitativa. Técnicas y procedimientos para desarrollar la Teoría Fundamentada. Universidad de Antioquia.

Tengblad, S. (2003). Classic, but not seminal: revisiting the pioneering study of managerial work. Scandinavian Journal of Management, 19(1), 85-101. https://doi.org/10.1016/ s0956-5221(01)00036-7

Tengblad, S. (2006). Is there a 'new managerial work'? A comparison with Henry Mintzberg's classic study 30 years later.Journal of management studies, 43(7), 14371461. https://doi.org/10.1111/j.1467-6486.2006.00651.x

Urrútia, G. \& Bonfill, X. (2010). Declaración PRISMA: Una propuesta para mejorar la publicación de revisiones sistemáticas y metaanálisis. Medicina Clínica, 135(11), 507-511. https://doi.org/10.1016/j.medcli.2010.01.015

Vuorikari, R., \& Castaño-Muñoz, J. (2016). Research Evidence on the Use of Learning Analytics - Implications for Education Policy. Joint Research Centre Science for Policy Report. European Commission. https://doi.org/10.2791/955210

Ward, M., \& Delamont, S. (2020). Introduction: using qualitative research methods for educational research. In M. Ward, \& S. Delamont. Handbook of Qualitative Research in Education, (pp. 1-4). Edward Elgar Publishing. https://doi. org/10.4337/9781788977159.00008

Wolcott, H. (2014). The shadow. Qualitative Research in Organizations and Management, 9(1), 2-2. https://doi.org/10.1108/QROM-07-2013-1164

World Bank. (2011). Learning for all: Investing in people's knowledge and skills to promote development. World Bank Group Education Sector Strategy 2020. World Bank. Retrieved from: https://bit.ly/38TAVS1

Fecha de recepción: 19 de enero de 2021.

Fecha de revisión: 10 de febrero de 2021.

Fecha de aceptación: 2 de julio de 2021. 